\title{
Polymorphisms of genes of the cardiac calcineurin pathway and cardiac hypertrophy
}

\author{
Odette Poirier ${ }^{1}$, Viviane Nicaud ${ }^{1}$, Theresa McDonagh ${ }^{2}$, Henry J Dargie ${ }^{2}$, Michel Desnos ${ }^{3}$, \\ Richard Dorent ${ }^{4}$, Gérard Roizès ${ }^{5}$, Ketty Schwartz ${ }^{6}$, Laurence Tiret ${ }^{1}$, Michel Komajda ${ }^{7}$ and \\ François Cambien*,1
}

\begin{abstract}
${ }^{1}$ INSERM U525, Epidemiologic and Molecular Genetics of Cardiovascular Diseases, Paris, France; ${ }^{2}$ Department of Cardiology and MRC Clinical Research Initiative in Heart Failure, Western Infirmary and University of Glasgow, UK; ${ }^{3}$ Hôpital Boucicaut, Paris, France; ${ }^{4}$ Service de Chirurgie Cardiaque, Groupe Hospitalier Pitié-Salpêtrière, Paris, France; ${ }^{5}$ CNRS UPR 1142, Montpellier, France; ${ }^{6}$ INSERM U523, Paris, France; ${ }^{7}$ Service de Cardiologie, Groupe Hospitalier Pitié-Salpêtrière, Paris, France
\end{abstract}

The study investigated the role of genetic polymorphisms in four genes of the calcineurin pathway on cardiac hypertrophy and dilated cardiomyopathy. The cardiac calcineurin pathway has been suggested to play a role in the development of cardiac hypertrophy in response to a number of physiological and pathological stimuli. Calcineurin, a heterodimeric protein composed of a catalytic and a regulatory subunit, activates the nuclear factor NFATC4 which after translocation to the nucleus associates with the transcription factor GATA4 to activate several cardiac genes involved in hypertrophic response. We have screened the genes encoding the four major components of the heart calcineurin pathway in 95 individuals and identified 27 polymorphisms. These polymorphisms were investigated in 400 selected subjects obtained from a population-based study (LOVE) in relation to echocardiographic parameters. A Gly/Ala substitution at position 160 of the NFATC4 protein (G160A) was associated with left ventricular mass and wall thickness $(P=0.02$ and 0.006 , respectively, $G A+A A$ vs $G G)$, the minor allele (Ala) being associated with lower mean values of these parameters. The other polymorphisms identified by the gene screen were not associated with cardiac phenotypes. For the G160A polymorphism in NFATC4, genotype frequencies were compared between patients with dilated cardiomyopathy and controls obtained from the CARDIGENE Study. Allele A carriers were less frequent in the patient than in the control group $(P=0.04)$. Although the strength of the associations was rather weak, these observations raise the hypothesis that the G160A polymorphism of the NFATC4 gene plays a role in the development of human cardiac hypertrophy. European Journal of Human Genetics (2003) 11, 659-664. doi:10.1038/sj.ejhg.5201023

Keywords: calcineurin; cardiac hypertrophy; genetic polymorphisms; association study

\section{Introduction}

Hypertrophic growth is an adaptative response of the heart to a variety of physiological and pathological stimuli,

*Correspondence: Dr F Cambien, INSERM U525, Epidemiologic and Molecular Genetics of Cardiovascular Diseases, Faculté de Médecine Pitié-Salpêtrière, 91 Boulevard de I'Hôpital, 75634 Paris cedex 13, France. Tel: +331 407796 39; Fax: +33 1407797 28;

E-mail: francois.cambien@chups.jussieu.fr

Received 5 December 2002; revised 17 March 2003; accepted 1 April 2003 including hypertension, myocardial infarction and endocrine disorders.

The hypertrophic response of cardiac myocytes to hemodynamic overload, mechanical stress or humoral factors is mediated by different signaling pathways. ${ }^{1}$ Experimental evidence suggests that calcium handling may play an important role in cardiac hypertrophy. ${ }^{2}$ In response to growth stimuli, cardiomyocytes increase their cytosolic $\mathrm{Ca}^{2+}$ level, which in turn activates calcineurin, a 
$\mathrm{Ca}^{2+} /$ calmodulin-dependent protein phosphatase. Calcineurin is a heterodimeric protein composed of a catalytic subunit, calcineurin A, also known as protein phosphatase 3 , catalytic subunit and a regulatory subunit, calcineurin B, also known as protein phosphatase 3, regulatory subunit. Whereas calcineurin B is encoded by a single gene (PPP3R1; OMIM \#601302), calcineurin A subunits can be coded by three different genes, respectively, PPP3CA (isoform alpha; OMIM \#114105), PPP3CB (isoform beta; OMIM \#114106) and PPP3CC (isoform gamma; OMIM \#114107). ${ }^{3}$ Molkentin et al ${ }^{4}$ reported that calcineurin plays a central role in the development of cardiac hypertrophy through activation by dephosphorylation of the nucleic factor of activated $\mathrm{T}$ cells (NFATC4). After activation, NFATC4 translocates to the nucleus where it associates with the transcription factor GATA-binding protein 4 (GATA4), to induce several cardiac genes involved in hypertrophic response. Given the potential importance of the calcineurin pathway in cardiac hypertrophy in humans, we designed this study to investigate the genetic variability of its major components PPP3CA, PPP3CB, NFATC4, GATA4 and its possible association with echocardiographic indexes of cardiac hypertrophy in a subsample of subjects participating in the Glasgow Heart Scan Study (LOVE Study). In addition, the NFATC4/G160A polymorphism was further investigated in the CARDIGENE Study to assess its possible association with dilated myocardiopathy.

\section{Methods}

\section{The LOVE Study}

The LOVE Study is a substudy of the Glasgow Heart Scan Study. This study has been previously described: ${ }^{5} 1600$ subjects (51\% women) aged $25-74$ years (mean age: $51 \pm 14$ years) from North Glasgow (UK), who had participated in the Third Glasgow MONICA Risk Factors Survey in 1992, ${ }^{6}$ were randomly selected. Participants underwent a standard echocardiography. Left ventricule mass (LVM) was indexed on body surface area and wall thickness (WT) was indexed on left ventricule telediastolic dimension. Ejection fraction (EF) was calculated by the biplane disc summation method (Simpson's rule). DNA was not available for 39\% of the subjects. For the LOVE Study, 400 subjects (180 men, 200 women, mean age: $45 \pm 13$ years, not taking cardiovascular drugs and whose body mass index (BMI) was $<28 \mathrm{~kg} / \mathrm{m}^{2}$ ), were selected in order to study the variation of LVM, WT and EF according to polymorphisms of candidate genes.

\section{The CARDIGENE Study}

This study has been already described. ${ }^{7}$ Briefly, 433 patients with dilated cardiomyopathy (DCM), aged 18-65 years, were recruited between 1994 and 1996 from 10 hospitals in France. The diagnosis was based on an ejection fraction $\leqslant 40$ and left ventricular dilation (end-diastolic volume $>140 \mathrm{ml} / \mathrm{m}^{2}$ on ventriculography or end-diastolic diameter $>34 \mathrm{~mm} / \mathrm{m}^{2}$ on echocardiography) confirmed over a 6-month period. All subjects had to be born in France and their parents had to be born in France or neighboring countries. Controls $(n=433)$ free of hypertension were recruited in Centres for Preventive Medicine all over metropolitan France and were matched for age, sex and region of birth. Their parents had to be born in France and their four grandparents had to be born in Europe. Each subject gave written informed consent.

\section{Identification of polymorphisms}

Genomic DNA was prepared from leukocytes. Polymorphisms discovery was performed in a sample of 95 individuals. In addition, in order to increase the power of detecting relatively rare variants that are associated with cardiac hypertrophy, these individuals were selected from among individuals in the Glasgow Heart Scan Study whose age-adjusted LVM (estimated by regression analysis) was in the upper decile of the sex-specific distribution.

DNA was amplified by polymerase chain reaction (PCR). All the primers used were designed to generate overlapping fragments of $<300 \mathrm{bp}$ spanning each exon of the four genes as well as their promoter and intronic sequences flanking exons (Table 1). The detection of sequence

Table 1 Description of the explored genes

\begin{tabular}{|c|c|c|c|c|c|c|c|c|c|c|}
\hline \multirow[b]{2}{*}{$\begin{array}{l}\text { OMIM gene } \\
\text { name }\end{array}$} & \multirow{2}{*}{$\begin{array}{l}\text { OMIM } \\
\text { accession } \\
\text { number }\end{array}$} & \multirow[b]{2}{*}{$\begin{array}{l}\text { Chromosome } \\
\text { location }\end{array}$} & \multirow[b]{2}{*}{$\begin{array}{l}\text { Number of } \\
\text { exons }\end{array}$} & \multicolumn{3}{|c|}{ Length explored (bp) } & \multirow{2}{*}{$\begin{array}{l}\text { Flanking } \\
\text { intronic } \\
\text { region }\end{array}$} & \multirow[b]{2}{*}{$3^{\prime} U T R$} & \multirow[b]{2}{*}{$3^{\prime}$ region } & \multirow[b]{2}{*}{$\begin{array}{l}\text { Analyzed-SSCP } \\
\text { fragments }\end{array}$} \\
\hline & & & & $5^{\prime}$ region & $5^{\prime} U T R$ & Exons & & & & \\
\hline PPP3CA & 114105 & $4 p 21-q 24$ & 14 & 324 & 148 & 1566 & 1735 & 543 & 0 & 16 \\
\hline РPР3СВ & 114106 & $10 q 21-q 22$ & 14 & 1091 & 117 & 1521 & 1919 & 214 & 0 & 17 \\
\hline GATA4 & 600576 & 8p23.1-p22 & 6 & 0 & 240 & 1324 & 949 & 199 & 0 & 16 \\
\hline NFATC4 & 602699 & $14 q^{11-2}$ & 10 & 544 & 141 & 2703 & 1213 & 53 & 94 & 18 \\
\hline
\end{tabular}

OMIM, online Mendelian Inheritance in Man (site available at: http://www3.ncbi.nlm.nih.gov/Omim/); PPP3CA, protein phosphatase 3, catalytic subunit, alpha isoform; PPP3CB, protein phosphatase 3, catalytic subunit, beta isoform; GATA4, GATA-binding protein 4; NFATC4, nuclear factor of activated T cells, cytoplasmic, 4; UTR, untranslated region of the exon. 
variants was performed using the single-strand conformation polymorphism (SSCP) analysis followed by direct sequencing of PCR fragments from patients with variable electrophoretic patterns.

\section{Genotyping}

For the -83 repeat in the PPP3CA gene, genotypes were deduced after an 8\% polyacrylamide denaturing gel electrophoresis (PAGE) of a radiolabeled PCR fragment. The polymorphisms $\mathrm{T}+74 / \mathrm{in} 1 \mathrm{C}$ and $\mathrm{T}+75 / \mathrm{in} 1 \mathrm{G}$ in the PPP3CA gene and A-131/in4G in the GATA4 gene could not be genotyped by our routine techniques because of their close proximity to another variant. All other polymorphisms were genotyped in the LOVE Study using radiolabeled allele-specific oligonucleotides (ASO). Primers, ASO, nomenclature of the polymorphisms and assay conditions are available on our web site (http://genecanvas.idf.inserm.fr).

\section{Statistical analysis}

The fit of genotype distributions to Hardy-Weinberg expectations was tested by a $\chi^{2}$ test with one degree of freedom (except for the repeat). Pairwise linkage disequilibrium coefficients (LDs) were calculated as reported as the ratio of the unstandardized coefficients to their minimal/maximal values $\left(\left|D^{\prime}\right|\right) .{ }^{8}$ A positive (negative) value indicates that the most frequent allele on locus 1 is preferentially linked to the most (least) frequent allele on locus 2. Haplotype frequencies were estimated using Arlequin software. ${ }^{9}$

Associations between genotypes and LVM, WT and EF were tested by ANOVA, adjusted for age and sex. Genotype/ phenotype associations were also tested assuming an additive effect of alleles, by entering the genotypes as a continuous variable (ie, common homozygotes $=0$, heterozygotes $=1$, minor homozygotes $=2$ ) in the model, and also by comparing all carriers of the minor allele $v s$ common homozygotes. To evaluate the possible impact of the combination of polymorphisms, all pairs of polymorphisms including their interaction term were tested in relation to echocardiographic variables using a General Linear Model. To evaluate further the potential role of the NFATC4/G160A polymorphism, its genotype frequencies were also compared between patients with DCM and controls in the CARDIGENE Study. The significance level used was $P<0.05$.

\section{Results}

\section{Description of gene polymorphisms}

The molecular screening of the four genes of the cardiac calcineurin pathway, performed on genomic DNA obtained from 95 independent individuals of European descent, resulted in the identification of 27 polymorphisms. The main characteristics of the investigated genes and sequenced fragments are summarized in
Table 1. Among the observed variants, six were located in the PPP3CA gene, two in the PPP3CB gene, twelve in the GATA4 gene, and seven in the NFATC4 gene (Table 2). All polymorphisms except three (for technical reasons) were genotyped in the LOVE Study. Complete description of the polymorphisms, linkage disequilibrium coefficients and haplotype frequencies for each gene can be found on our web site (http://genecanvas.idf.inserm.fr). All observed genotype distributions were compatible with HardyWeinberg equilibrium. Two polymorphisms, PPP3CA/ A83A and GATA4/N352N, detected by the gene screens, were not present in the LOVE Study, probably because they were rare.

The three repeats allele of the trinucleotides repeat polymorphism at position -83 in the $5^{\prime}$ UTR region of the PPP3CA gene was in complete negative LD with PPP3CA/ G-193T and PPP3CA/L365L. The two polymorphisms found in PPP3CB were in complete negative LD, resulting in only three haplotypes. Among the 12 polymorphisms found in the GATA4 gene, two resulted in an amino-acid change (GATA4/S377G and GATA4/A411 V, with minor allele frequencies of 0.11 and $<0.01$, respectively). The GATA4 gene polymorphisms were in strong $\mathrm{LD}$, the values of all pairwise LD coefficients but one being in the range $0.8-1$. In the second exon of the NFATC4 gene, an insertion/deletion polymorphism with minor allele frequency of 0.03 predicted the presence/absence of three amino acids (Gly-Gly-Ala) at position 103 of the encoded protein (AA103del/ins) and another polymorphism predicted a Gly/Ala substitution at position 160 (G160A). The NFATC4/G160A polymorphism was nearly completely concordant with the NFATC4/T607 T polymorphism ( $<5 \%$ recombinant), and was in strong positive LD (between 0.8 and 1) with all other NFATC4 gene polymorphisms.

\section{Associations of gene polymorphisms with echocardiographic parameters}

In the LOVE Study, NFATC4/G160A was the only polymorphism associated with indexes of cardiac hypertrophy. Lower mean LVM and WT were observed in carriers of the NFATC4/A160 allele (Table 3). The association was statistically significant (comparing GG vs GA+AA, $P=0.02$ and 0.006 for LVM and WT respectively), and the test of homogeneity between men and women was not statistically significant. Further adjustment on BMI, smoking and high physical activity did not affect the results. The mean EF was similar across NFATC4/G160A genotypes in both sexes. Association between cardiac echographic parameters and pairs of polymorphisms were also tested, but revealed no significant synergistic effect. To evaluate its possible clinical relevance, the NFATC4/G160A polymorphism was investigated in patients with DCM (336 men and 84 women) and controls (345 men and 88 women) participating in the 
Table 2 Description of the polymorphisms

\begin{tabular}{|c|c|c|c|c|}
\hline Gene & Polymorphism & Position & Type of variation & Minor allele frequency \\
\hline \multirow[t]{5}{*}{ РPР3СA } & $\begin{array}{l}\mathrm{G}-193 \mathrm{~T} \\
-83 \mathrm{rpt}^{\mathrm{a}}\end{array}$ & $\begin{array}{l}5^{\prime} \text { region } \\
5^{\prime} \text { UTR }\end{array}$ & $\begin{array}{l}\mathrm{G} / \mathrm{t} \\
\text { CGG repeat } \\
\text { (from one copy } \\
\text { up to four repeats) }\end{array}$ & $\begin{array}{l}0.02 \\
1=0.69 / 2=0.30 / 3=0.01 / 4=0.0025\end{array}$ \\
\hline & $\mathrm{T}+74 /$ in1C (74th nucleotide of intron 1 ) & Intron 1 & $\mathrm{~T} / \mathrm{c}$ & Not genotyped \\
\hline & $\mathrm{T}+75 /$ in $1 \mathrm{G}$ (75th nucleotide of intron 1 ) & Intron 1 & $\mathrm{~T} / \mathrm{g}$ & Not genotyped \\
\hline & A83A & Exon 2 & $\mathrm{G} / \mathrm{a}$ & Not found in the LOVE Study \\
\hline & L365L & Exon 10 & $\mathrm{G} / \mathrm{c}$ & 0.02 \\
\hline \multirow[t]{2}{*}{ РPР3СВ } & $\mathrm{G}+10 /$ in7C (10th nucleotide of intron 7 ) & Intron 7 & $\mathrm{G} / \mathrm{C}$ & 0.03 \\
\hline & $\mathrm{G}+81 \mathrm{~A}$ & $3^{\prime}$ UTR & $\mathrm{G} / \mathrm{a}$ & 0.02 \\
\hline \multirow[t]{12}{*}{ GATA4 } & T233T & Exon 2 & $\mathrm{G} / \mathrm{a}$ & $<0.01$ \\
\hline & $\mathrm{C} 274 \mathrm{C}$ & Exon 3 & $\mathrm{C} / \mathrm{t}$ & $<0.01$ \\
\hline & $A+56 /$ in $4 C$ (56th nucleotide of intron 4 ) & Intron 4 & $\mathrm{~A} / \mathrm{C}$ & 0.44 \\
\hline & $\begin{array}{l}180 / \text { in } 4 / \text { ins/del (180th nucleotide before } \\
\text { exon } 5 \text { ) }\end{array}$ & Intron 4 & $\pm \mathrm{A}$ & 0.31 \\
\hline & A-139/in4T (139th nucleotide before exon 5) & Intron 4 & $\mathrm{~A} / \mathrm{t}$ & 0.17 \\
\hline & A-131/in4G (131st nucleotide before exon 5) & Intron 4 & $\mathrm{~A} / \mathrm{g}$ & Not genotyped \\
\hline & T-128/in4C (128th nucleotide before exon 5) & Intron 4 & $\mathrm{~T} / \mathrm{c}$ & 0.41 \\
\hline & C-115/in4T (115th nucleotide before exon 5) & Intron 4 & $\mathrm{C} / \mathrm{t}$ & 0.28 \\
\hline & N352N & Exon 5 & $\mathrm{C} / \mathrm{t}$ & Not found in the LOVE Study \\
\hline & S377G & Exon 5 & $A / g$ & 0.11 \\
\hline & G-108/in5A (108th nucleotide of intron 5) & Intron 5 & $\mathrm{G} / \mathrm{a}$ & 0.46 \\
\hline & A411V & Exon 6 & $\mathrm{C} / \mathrm{t}$ & $<0.01$ \\
\hline \multirow[t]{7}{*}{ NFATC4 } & $-586 \mathrm{del} / \mathrm{ins}$ & $5^{\prime}$ region & \pm GGA & 0.23 \\
\hline & AA103del/ins & Exon 2 & Insertion of three amino & 0.03 \\
\hline & G160A & Exon 2 & $\mathrm{C} / \mathrm{g}$ & 0.42 \\
\hline & D187D & Exon 2 & $\mathrm{C} / \mathrm{t}$ & 0.31 \\
\hline & T607T & Exon 6 & $\mathrm{C} / \mathrm{t}$ & 0.41 \\
\hline & G700G & Exon 9 & $\mathrm{~T} / \mathrm{C}$ & 0.06 \\
\hline & $\mathrm{G}+41 \mathrm{~T}$ & $3^{\prime}$ UTR & $\mathrm{G} / \mathrm{t}$ & 0.22 \\
\hline
\end{tabular}

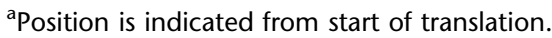

Table 3 Echocardiographic parameters (mean, SEM) according to NFATC4/G160A polymorphism in the LOVE Study

\begin{tabular}{llll}
\hline & $\begin{array}{l}\text { Left } \\
\text { ventricule mass } \\
\text { Mean } \\
\text { (SEM) }\end{array}$ & $\begin{array}{l}\text { Wall } \\
\text { thickness } \\
\text { Mean } \\
\text { (SEM) }\end{array}$ & $\begin{array}{l}\text { Ejection } \\
\text { fraction } \\
\text { Mean } \\
\text { (SEM) }\end{array}$ \\
\hline GG, $n=123$ & $74.8(1.1)$ & $0.679(0.013)$ & $47.6(0.7)$ \\
GA, $n=179$ & $71.6(0.9)$ & $0.641(0.010)$ & $47.0(0.6)$ \\
AA, $n=63$ & $71.8(1.6)$ & $0.622(0.018)$ & $47.0(0.9)$ \\
ANOVA (2 DF) & $P=0.068$ & $P=0.016$ & $P=0.82$ \\
$\begin{array}{l}\text { Additive effect } \\
\text { (1 DF) }\end{array}$ & $P=0.054$ & $P=0.005$ & $P=0.59$ \\
$\begin{array}{l}\text { Test GG vs GA+AA } \\
\text { (1 DF) }\end{array}$ & $P=0.020$ & $P=0.006$ & $P=0.52$ \\
\hline
\end{tabular}

Means were adjusted for age and sex.

CARDIGENE Study (Table 4). Carriers of the GA or AA genotypes were less frequent in DCM patients than in controls $(P=0.04)$, and the test of homogeneity between men and women was not statistically significant.
The gender ratio, the percentage of smokers, and the mean age, BMI and daily alcohol consumption were similar in the 3 NFATC4/G160A genotypes in patients with DCM and controls. In the CARDIGENE Study, 224 patients with DCM had received a heart transplantation and 196 had not. In nontransplanted patients, the GG/GA/AA genotype frequencies were $37.3,45.9$ and $16.8 \%$, respectively, whereas in the transplanted patients they were $31.2,43.3$ and $25.5 \%$, respectively $(P=0.032$ for the GG+GA vs AA comparison). In both groups of DCM patients, heterozygote individuals where less frequent than in control subjects (51.7\%); however in nontransplanted patients, AA homozygotes were also less common (16.8\%) than in controls $(20.6 \%)$, whereas the reverse was observed in transplanted patients $(25.5 \%)$.

\section{Discussion}

The objective of this study was to test the possible implication of polymorphisms of genes encoding compo- 
Table 4 Frequency (\%) of NFATC4/G160A genotypes in cases with dilated cardiomyopathy and controls of the CARDIGENE Study

\begin{tabular}{|c|c|c|c|c|c|c|c|}
\hline & & & hotype & & & & \\
\hline & $N$ & $(\%)$ & $N$ & (\%) & $N$ & $(\%)$ & Allele A frequency \\
\hline DCM cases $n=420$ & 143 & (34.1) & 187 & $(44.5)$ & 90 & $(21.4)$ & 0.44 \\
\hline Controls $n=433$ & 120 & (27.7) & 224 & $(51.7)$ & 89 & (20.6) & 0.46 \\
\hline
\end{tabular}

Odds ratio $(95 \% \mathrm{Cl})$ for dilated cardiomyopathy, adjusted for age and sex, associated with allele A carrying $(\mathrm{GA}+\mathrm{AA}): 0.74[0.55-0.99] P=0.040$.

nents of the heart calcineurin pathway in cardiac hypertrophy. Investigating the polymorphism of several genes in the same pathway in relation to candidate phenotypes, as we already did for the Receptor of Advanced Glycation products, ${ }^{10}$ is based on the premise that the genetics of the whole system is more relevant to the phenotype than the variability of each gene considered separately as a consequence of the possible additive or interactive effects on the phenotype of the polymorphisms of the different genes. In this particular study however, despite the investigation of a fairly large number of polymorphisms, only one among the four nonsynonymous polymorphisms, NFATC4/G160A, was associated with the investigated phenotype and combined analysis of the different polymorphisms did not suggest any synergistic effect of the different variants on cardiac echocardiographic parameters. Overall, the results show that compared to noncarriers, carriers of the NFATC4/A160 allele have lower mean LVM and WT in the LOVE Study and are less frequently observed in patients with DCM than in controls in the CARDIGENE Study. This may suggest that the A160 allele protects against cardiac hypertrophy. The observation that the difference between DCM patients and controls was principally because of a lower frequency of heterozygotes in patients, while AA160 homozygotes were as frequent in cases as in controls is intriguing. However, the increased frequency of homozygosity for the putatively protective allele in heart-transplanted patients when compared to controls, whereas the reverse was observed in nontransplanted patients may reflect a survival bias favoring carriers of the AA160 genotype in transplanted patients. This hypothesis might be tested in an appropriate prospective setting. Our results are only suggestive of a potential beneficial effect of the A160 allele; indeed, as a large number of polymorphisms were detected, the number of statistical tests performed was accordingly large; in addition, the associations reported with the NFATC4/ G160A polymorphism while coherent, are rather weak and should therefore be tested in other appropriately designed studies of relevant groups of patients. A major limitation of this study is related to the particular groups of individuals in whom the genetic polymorphisms were investigated and to the measured phenotypes. The Glasgow Heart Scan
Study and the LOVE Substudy were designed to provide an epidemiological description of cardiac echocardiographic parameters in the population at large, ${ }^{5}$ this may not be the most appropriate setup for investigating genetic factors that might contribute to cardiac hypertrophy. The polymorphisms of the cardiac calcineurin pathway, in particular those encoding protein polymorphisms or located in regulatory regions of the genes should also be investigated in patients with other types of heart disease than DCM and in situations where they could be response modifiers of cardiac phenotypes, for example, in patients with genetic disorders of the myocardium, in patients with a recent myocardial infarction or in response to physical training.

The sequence of the NFATC4 protein is highly conserved between mouse and human. The G160A polymorphism is located within a stretch of 74 aminoacids that are common to both species. The YREAG[A|G]QGGGA sequence that encompasses the G160A polymorphism is identical in human and mouse, with a $G$ at position 160 in the mouse. Despite this identity, 10 out of the 11 triplets of nucleic acids encoding this sequence differ between both species. This is likely the consequence of a strong resistance of the protein sequence to evolutionary change, while synonymous changes of the gene sequence are well tolerated. Concerning codon 160, it may be proposed that either GGC or GGT are the ancestral form to both human and mouse whereas the G/C mutation that generated the GCC (Alanine) codon occurred in the human lineage. While the evolutionary arguments above may suggest that the region of the NFATC4 gene encompassing the G160A polymorphism is functionally important, the context in which this functionality is manifest and the specific consequences of the polymorphism are unknown. Members of the NFAT protein family are ubiquitous regulators of cell differentiation and adaptation, ${ }^{11}$ they possess several domains responsible for binding with calcineurin, DNA binding, interaction with transcription factors such as AP-1, GATA or PPAR $\gamma$, translocation in and out of the nucleus, or for the action of phosphatases; ${ }^{12}$ it appears that the G160A polymorphism is not located within one of these functional domains. The other polymorphisms reported in Table 2 were also examined in terms of their putative functionality based on in silico analysis. The nonsynon- 
ymous substitutions in the GATA4 gene S377G and A411V are rather conservative changes based on the Blossom 62 substitution matrix; ${ }^{13}$ the G-193 T polymorphism in the promoter region of the PPP3CA gene is not located in a known consensus sequence and there is no a priori evidence that the different intronic polymorphisms found in the PPP3CA, PPP3CB and GATA4 genes may affect splicing.

In conclusion, we have screened four genes encoding the major components of the the calcineurin pathway in the heart and identified 27 polymorphisms. One of them, G160A located in the NFATC4 gene is associated with echocardiographic parameters of cardiac hypertrophy and with DCM. This observation raises the hypothesis that this polymorphism may play a role in the development of human cardiac hypertrophy.

\section{Acknowledgements}

The Glasgow Heart Scan Study was funded by the Chief Scientist Office of the Scottish Home and Health Department.

\section{References}

1 Molkentin JD, Dorn G: Cytoplasmic signaling pathways that regulate cardiac hypertrophy. Annu Rev Physiol 2001; 63: 391-426.

2 Balke CW, Shorofsky SR: Alterations in calcium handling in cardiac hypertrophy and heart failure. Cardiovasc Res 1998; 37: 290-299.

3 Wang MG, Yi H, Guerini D, Klee CB, McBride OW: Calcineurin A alpha (PPP3CA), calcineurin A beta (PPP3CB) and calcineurin B (PPP3R1) are located on human chromosomes 4, 10q21 $\rightarrow$ q22 and 2p16 $\rightarrow$ p15 respectively. Cytogenet Cell Genet 1996; 72: 236-241.

4 Molkentin JD, Lu JR, Antos CL et al: A calcineurin-dependent transcriptional pathway for cardiac hypertrophy. Cell 1998; 93: 215-228.

5 McDonagh TA, Morrison CE, Lawrence A et al: Symptomatic and asymptomatic left-ventricular systolic dysfunction in an urban population. Lancet 1997; 350: 829-833.

6 Tunstall-Pedoe H, Kuulasmaa K, Amouyel P, Arveiler D, Rajakangas AM, Pajak A. Myocardial infarction and coronary deaths in the World Health Organization MONICA Project.
Registration procedures, event rates, and case-fatality rates in 38 populations from 21 countries in four continents. Circulation 1994; 90: 583-612.

7 Tiret L, Mallet C, Poirier O et al: Lack of association between polymorphisms of eight candidate genes and idiopathic dilated cardiomyopathy: the CARDIGENE study. J Am Coll Cardiol 2000; 35: 29-35.

8 Tiret L, Amouyel P, Rakotovao R, Cambien F, Ducimetiere P: Testing for association between disease and linked marker loci: a log-linear-model analysis. Am J Hum Genet 1991; 48: 926-934.

9 Schneider S, Roessli D, Excoffier L: Arlequin: a software for population genetics data analysis. Ver 2.000. Genetics and Biometry Lab, Department of Anthropology, University of Geneva, 2000.

10 Poirier O, Nicaud V, Vionnet $\mathrm{N}$ et al: Polymorphism screening of four genes encoding advanced glycation end-product putative receptors. Association study with nephropathy in type 1 diabetic patients. Diabetes 2001; 50: 1214-1218.

11 Horsley V, Pavlath GK: NFAT: ubiquitous regulator of cell differentiation and adaptation. J Cell Biol 2002; 156: 771-774.

12 Crabtree GR: Generic signals and specific outcomes: signaling through Ca2+, calcineurin, and NF-AT. Cell 1999; 96: 611-614.

13 Henikoff S, Henikoff JG: Amino acid substitution matrices from protein blocks. Proc Natl Acad Sci USA 1992; 89: 10915-10919.

\section{Appendix}

CARDIGENE Project Management Group: M Desnos, Service de Cardiologie, Hôpital Boucicaut, Paris; R Dorent, Service de Chirurgie Cardiaque, Groupe Hospitalier Pitié-Salpêtrière, Paris; M Komajda, Service de Cardiologie, Groupe Hospitalier Pitié-Salpêtrière, Paris; G Roizès, INSERM U249, Montpellier; K Schwartz, INSERM U523, Paris; L Tiret, INSERM U525, Paris, France.

CARDIGENE recruitment centers: CHU Ambroise Paré, Boulogne Billancourt (O Dubourg); CHU Henri Mondor, Créteil (F Paillart); CHR de Lille (A Millaire); Hôpital Louis Pradel, Lyon (X André-Fouët, J Beaune, P Touboul); CHR de Nancy (Y Juillière); CHR de Nantes (J-B Bouhour, N Chedru); CHU Pitié-Salpêtrière, Paris (R Dorent, M Komajda); Hôpital Boucicaut, Paris (M Desnos); Hôpital Bichat, Paris (M-C Aumont); CHR de Strasbourg (A Sacrez). 